\title{
Maternal psychosocial risk factors and offspring gestational epigenetic age acceleration in a South African birth cohort study
}

Running title: Psychosocial risk and epigenetic age acceleration

Nastassja Koen ${ }^{\mathrm{a}, \mathrm{b}, \mathrm{c}}(\mathrm{PhD})$, Meaghan Jones ${ }^{\mathrm{d}}(\mathrm{PhD})$, Raymond T. Nhapie (BBusSci), Kirsten A. Donald ${ }^{c, f}(\mathrm{PhD})$, Whitney Barnett $(\mathrm{MPH})$, Nadia Hoffman ${ }^{\mathrm{a}}(\mathrm{MA})$, Julia L. Maclsaac ${ }^{\mathrm{h}}(\mathrm{PhD})$, Alexander M. Morin ${ }^{\mathrm{h}}$, David T.S. Lin ${ }^{\mathrm{h}}(\mathrm{PhD})$, Michael S. Kobor ${ }^{\mathrm{h}, \mathrm{ij}}(\mathrm{PhD})$, Karestan C. Koenen $^{k, l}(\mathrm{PhD})$, Heather J. Zar ${ }^{\mathrm{g}}(\mathrm{PhD})$, Dan J. Stein ${ }^{\mathrm{a}, \mathrm{b}, \mathrm{c}}(\mathrm{PhD})$

${ }^{a}$ Department of Psychiatry and Mental Health, University of Cape Town, South Africa

${ }^{\mathrm{b}}$ South African Medical Research Council (SAMRC) Unit on Risk and Resilience in Mental Disorders, Cape Town, South Africa

${ }^{c}$ Neuroscience Institute at the University of Cape Town, Faculty of Health Sciences, University of Cape Town, South Africa

${ }^{d}$ Biochemistry and Medical Genetics, Max Rady College of Medicine, Rady Faculty of Health Sciences, University of Manitoba, Manitoba, Canada

e Department of Paediatrics and Child Health, Red Cross War Memorial Children's Hospital, University of Cape Town, Cape Town, South Africa

${ }^{f}$ Division of Developmental Paediatrics, Department of Paediatrics and Child Health, Red Cross War Memorial Children's Hospital, University of Cape Town, South Africa

${ }^{9}$ Department of Paediatrics \& Child Health and SAMRC Unit on Child and Adolescent Health, University of Cape Town, South Africa

${ }^{\mathrm{h}}$ Department of Medical Genetics, Centre for Molecular Medicine and Therapeutics, BC Children's Hospital Research Institute, University of British Columbia, Vancouver, Canada

' BC Children's Hospital Research Institute, Vancouver, Canada

j Canadian Institute for Advanced Research, Canada

${ }^{k}$ Department of Epidemiology, Harvard T.H. Chan School of Public Health, Boston, MA, USA

'Psychiatric and Neurodevelopmental Genetics Unit and Department of Psychiatry, Massachusetts General Hospital, Boston, MA, USA 


\section{ABSTRACT}

Epigenetic age (EA) acceleration is associated with higher risk of chronic disease and mortality in adults. However, little is known about whether and how in utero exposures might shape gestational EA acceleration at birth. We aimed to explore associations between maternal psychosocial risk factors and offspring gestational EA acceleration at birth in a South African birth cohort study - the Drakenstein Child Health Study. Maternal psychosocial risk factors included trauma/stressor exposure; posttraumatic stress disorder (PTSD); depression, psychological distress; and alcohol/tobacco use. Offspring gestational EA acceleration at birth was calculated using an epigenetic clock previously devised for neonates. Bivariate linear regression was used to explore unadjusted associations between maternal risk factors and offspring gestational EA acceleration at birth. A stepwise regression method was then used to determine the best multivariable model for adjusted associations. Data from 272 maternal-offspring dyads were included in the current analysis. In the stepwise regression model, maternal trauma exposure $(\beta=7.92 ; p<0.01)$ or PTSD $(\beta=7.46 ; p<0.01)$ were significantly associated with offspring gestational EA acceleration at birth, controlling for ethnicity, offspring sex, head circumference at birth, maternal HIV status, and prenatal tobacco or alcohol use. In site-stratified models, these associations retained statistical significance and direction of effect. Maternal trauma exposure or PTSD may thus be associated with offspring gestational EA acceleration at birth. Given the novelty of this preliminary finding, and its potential translational relevance, further studies to delineate underlying biological pathways and to explore clinical implications of EA acceleration are warranted. 


\section{INTRODUCTION}

Maternal exposure to psychosocial risk factors during pregnancy - including traumatic stressors, psychiatric disorders and symptoms, and substance misuse - constitute a notable public health concern; and may be associated with poor birth outcomes and adverse health and development in affected offspring ${ }^{1,2}$. Given the high burden of psychosocial risk factors in low- and middle-income countries (LMICs) such as South Africa ${ }^{3}$, maternal-offspring dyads in these settings are particularly vulnerable. Prenatal psychosocial risk exposure may be associated with measurable changes in DNA methylation (DNAm) at specific genomic sites in the affected offspring ${ }^{4}$. It is noteworthy, though, that a large meta-analysis found no large epigenome-wide effects of prenatal maternal stress exposure on offspring differential DNAm ${ }^{5}$. Recent work has also investigated DNAm as a predictor of chronological age in children and adults 6,7 . However, given the limitations in transferability of such 'epigenetic clocks' (which were calibrated initially for adult populations); specialised clocks have recently been generated to estimate gestational age at birth ${ }^{8-10}$.

Epigenetic age (EA) acceleration - greater DNAm gestational age relative to chronological age - has been found to be associated with increased risk of age-related disorders and mortality in adults ${ }^{11,12}$. Further, recent work has reported significant associations between a number of maternal-offspring biomedical factors (eg. pre-pregnancy maternal obesity; maternal age over 40 years at delivery; lower offspring birthweight; lower 1-minute Apgar score) and EA acceleration at birth in the affected offspring ${ }^{13,14}$. However, EA deceleration (ie. lower epigenetic age relative to chronological age) associated with maternal risk (eg. insulin-treated gestational diabetes mellitus) has also been reported ${ }^{13}$. Thus, the functional implications of epigenetic age acceleration/deceleration remain unclear ${ }^{13,15}$. There is also a paucity of existing literature exploring maternal psychosocial predictors of offspring gestational EA acceleration, particularly in LMICs. 
This analysis was nested within the Drakenstein Child Health Study (DCHS), an ongoing, interdisciplinary, longitudinal birth cohort study investigating determinants of maternal-child health in a poor, peri-urban South African community ${ }^{16-18}$. We have previously reported a high burden of maternal psychosocial risk factors (eg. traumatic stress exposure/posttraumatic stress disorder (PTSD), alcohol and tobacco use); and significant associations between these factors and adverse offspring birth and neurodevelopmental outcomes in this cohort ${ }^{19-23}$. In the current analysis, we aimed to investigate associations between maternal psychosocial risk factors and offspring EA acceleration at birth. We hypothesised that key maternal risk factors would be significantly associated with offspring gestational EA acceleration in this study sample.

\section{MATERIALS/SUBJECTS AND METHODS}

\section{Participants}

Pregnant women were recruited at 20-28 weeks' gestation from two primary care clinics in the Drakenstein sub-district in Paarl, Western Cape, South Africa - one (TC Newman) serves a predominantly Mixed ancestry community; while the other (Mbekweni) serves primarily a Black/African ancestry community. Within the DCHS, mothers are followed throughout pregnancy and childbirth until the index child is at least 5 years old ${ }^{16-18}$. General inclusion and exclusion criteria for the DCHS are described fully elsewhere ${ }^{17}$.

For this analysis, only samples of mothers with complete and accurate sociodemographic and psychosocial phenotype data; with recorded maternal informed consent for the collection, storage, and future analyses of offspring DNA; and with non-contaminated offspring Illumina Infinium HumanMethylation450K or MethylationEPIC BeadChip data were eligible for inclusion ${ }^{24}$ (see also Statistical Analyses, below). 
This study sample included mothers enrolled into the larger study between March 2012 and March 2015. From a total of 1225 mothers enrolled, 1143 were retained in the cohort at the time of birth; and delivered live births. From this sub-sample, 276 mothers had offspring with non-contaminated HumanMethylation450K $(n=120)$ or MethylationEPIC $(n=156)$ BeadChip data; and complete maternal/offspring phenotype data. This sub-sample was initially selected for analysis based on a number of risk criteria of relevance to the larger DCHS including (but not limited to) maternal exposure to psychological trauma and/or PTSD. Two sets of twins was excluded, due to concerns of analytic mixed effects. Thus, data from a sub-sample of 272 maternal-offspring dyads were included in the current analysis.

\section{$\underline{\text { Variables and Measurement }}$}

Maternal sociodemographic and antenatal psychosocial risk factors; offspring anthropometric outcomes; and offspring gestational EA acceleration at birth were assessed, as is detailed below. Maternal psychosocial assessment in the DCHS has been described fully previously ${ }^{17}$.

\section{Maternal sociodemographic characteristics}

Socioeconomic status (SES) and related sociodemographic characteristics were assessed using a questionnaire adapted from the South Africa Stress and Health Study $(\mathrm{SASH})^{25}$.

\section{Maternal antenatal psychosocial risk factors}

Exposure to stressful events - either recently or in one's lifetime - was assessed using the modified World Mental Health Life Events Questionnaire (LEQ; adapted for our purposes from the SASH ${ }^{25}$; the Childhood Trauma Questionnaire (CTQ) ${ }^{26}$; and the Intimate Partner Violence (IPV) Questionnaire (adapted from the WHO Multicountry Study ${ }^{27}$ and the Women's Health Study (Zimbabwe) $)^{28}$. The Modified PTSD Symptom Scale (MPSS) ${ }^{29}$ was used as a rapid screening for psychological trauma exposure and/or PTSD, based on the DSM diagnostic criteria and adapted for our purposes to incorporate a more "broad" 
approach to defining the index traumatic events (eg. see Stein et al. $2014^{30}$ for "narrow" vs "broad" approaches for diagnosing PTSD). The MPSS screens for PTSD symptoms in the preceding 2 weeks. Psychological distress was assessed using the Self-Reporting Questionnaire (SRQ-20) $)^{31,32}$; and depression using the Beck Depression Inventory II (BDIII) ${ }^{33-35}$ and the Edinburgh Postnatal Depression Scale (EPDS) ${ }^{36,37}$. Maternal substance misuse was identified using the World Health Organization's (WHO's) Alcohol, Smoking and Substance Involvement Screening Test (ASSIST) ${ }^{38-40}$ and a retrospective Alcohol Exposure Questionnaire (AEQ). The AEQ was designed for the purposes of the DCHS to quantify alcohol use (during any of the three trimesters of pregnancy) in mothers assessed as highrisk on the ASSIST.

\section{Offspring anthropometry at birth}

Offspring weight, head circumference, and height/length at birth were measured by trained clinical staff, and the relevant $z$ scores were then calculated using the Fenton preterm growth charts ${ }^{41,42}$. Following the WHO's convention, low weight-for-age $z$ score (WAZ), low head circumference-for-age z score (HCAZ) and low height-for-age z score (HAZ) each was defined as a score of 2 standard deviations or more below the mean ${ }^{43}$. Prematurity was defined as birth before 37 completed weeks' gestation; and low birthweight as less than $2,500 \mathrm{~g}$.

\section{Umbilical cord blood collection}

In the DCHS, umbilical cord blood was collected by trained staff after offspring delivery but before delivery of the placenta. The cord was clamped and cut, after which the clamp was released and cord blood drained by gravity into a kidney dish. Thereafter, cord blood was collected using a syringe for processing and storage ${ }^{24}$. Staff were specifically trained to ensure only cord blood drained into the collection dish and that to their best ability, no other/external blood was collected. 


\section{Ethical Considerations}

The DCHS was approved by the Human Research Ethics Committee (HREC) of the Faculty of Health Sciences, University of Cape Town (UCT) and by the Western Cape Provincial Research Committee. Within the DCHS, all potential participants were provided with a consent form describing the scope and aims of the study, in their preferred language (English, Afrikaans or isiXhosa). Following verbal and written informed consent, mothers were asked to complete a battery of self-report measures at an antenatal study visit (28-32 weeks' gestation). While maternal phenotype data from a number of postnatal timepoints are also collected within the larger $\mathrm{DCHS}^{16,17}$; the current analysis pertains to the antenatal maternal assessment only.

All measures were administered by trained study fieldworkers in either English, Afrikaans or isiXhosa, as per participants' preferences. Interviews were conducted in private onsite consultation rooms and every effort was made by study staff to ensure confidentiality. Participants were also provided with refreshments and standard reimbursement for transport costs. On completion of the study assessment, those participants with suspected psychopathology or psychosocial risk (eg. trauma/IPV exposure, PTSD, depression and/or substance use) were referred by study staff to the most appropriate care providers in the community, according to a standard operating procedure devised for the purposes of the DCHS. Health promotion information leaflets designed by the study team (and translated into participants' preferred language) were also offered to all enrolled mothers, and included contact details for local and accessible health service providers. 


\section{Statistical Methods}

Data were analysed using $\mathrm{R}^{44}$ and STATA ${ }^{45}$.

\section{Maternal-offspring phenotype}

Frequency distributions and medians (interquartile ranges) were used to describe maternal sociodemographic characteristics and psychosocial risk profile; as well as infant anthropometric parameters at birth.

\section{Offspring epigenetic age acceleration}

As has been described fully previously ${ }^{24}$, offspring DNAm was measured using the Illumina Infinium HumanMethylation450K (Illumina, San Diego, USA) or the MethylationEPIC BeadChip ${ }^{46}$ as per manufacturers' instructions. Raw data were imported into Illumina GenomeStudio Software for background subtraction and colour correction, and then exported for processing using the lumi package in R (version 3.2.3). Quality control procedures were standardised and are discussed in detail elsewhere ${ }^{24}$.

Offspring gestational EA acceleration at birth was calculated using an epigenetic clock particularly suited for newborns which outputs gestational age $\mathrm{e}^{8,10}$ and is based on prior work in adult populations using chronological age ${ }^{6}$. This method employs a panel of $\mathrm{CpGs}$ to predict chronological age, of which 8 are missing on the EPIC array. For EPIC data, these 8 CpGs were included with values set to NA. In the current analysis, gestational EA acceleration was calculated as the residuals of the linear model between epigenetic gestational age and chronological $\mathrm{GA}^{47}$. 


\section{Associations between maternal psychosocial risk factors and offspring gestational epigenetic age acceleration at birth}

Bivariate analyses were used to explore unadjusted associations between key maternal sociodemographic and psychosocial risk variables (eg. income, trauma/stress, PTSD, psychological distress, depression, tobacco/alcohol use and maternal HIV infection) and offspring gestational EA acceleration at birth. Thereafter, a stepwise model selection method was used to obtain the best multiple regression model (from which the adjusted associations were obtained), based on minimizing the Akaike Information Criterion (AIC). Likelihood ratio tests were then used to eliminate any statistically insignificant variables from this model. Given prior site-specific differences in key exposure variables such as tobacco/alcohol use ${ }^{17}$ and maternal HIV (see below), the regression analysis was repeated, stratified by study site.

\section{RESULTS}

\section{Maternal sociodemographic characteristics}

This study sample comprised 151 (56\%) women recruited from Mbekweni and 121 (44\%) from TC Newman. At enrolment, the median (IQR) age of mothers was approximately 26 $(22 ; 31)$ years, Table 1 . Approximately a third were primigravid, and most $(61 \%)$ were unmarried/not co-habiting with their partner. Despite approximately half of the study sample having completed some secondary education, nearly three quarters $(73 \%)$ were unemployed and the vast majority (84\%) reported a monthly household income of $\leq$ ZAR $5000(\approx U S D$ 400). While the prevalence of maternal HIV infection in the study sample was $24 \%$, a statistically significant site-specific difference was noted $(p<0.01)$, with $6 \%$ of mothers at TC Newman HIV infected, versus $38 \%$ at Mbekweni. No offspring in this study sample was HIV infected. 


\section{Maternal antenatal psychosocial risk}

Most mothers $(61 \%)$ in this study sample reported exposure to any psychological trauma during their lifetimes; $42 \%$ had been exposed to trauma (abuse/neglect) during childhood; and the prevalence of lifetime exposure to intimate partner violence (IPV) was 54\% (with $43 \%$ having experienced IPV during the past year), Table 1. The prevalence of current PTSD was $31 \%$. Despite a relatively low median (IQR) score on the modified Life Events Questionnaire [2 (1; 3)], 29\% of the study sample reporting symptoms of psychological distress on the SRQ-20. Similarly, almost a third scored above threshold for depression on both the BDI-II (31\%) and the EPDS (30\%). Alcohol consumption during pregnancy was reported in $17 \%$ of participants, and prenatal tobacco use in $29 \%$. A notable and statistically significant site-specific difference was evident in the latter, with $9 \%$ of mothers from Mbekweni and 53\% from TC Newman having smoked tobacco during pregnancy $(p<0.01)$.

\section{Offspring anthropometry at birth}

More than half $(56 \%)$ of offspring in this study sample was male; the median (IQR) gestational age at birth of $39(38 ; 40)$ weeks, Table 2. Almost a fifth of offspring $(19 \%)$ were born pre-term; 11\% had low birth weight; $8 \%$ had decreased weight-for-age z (WAZ) scores at birth and $12 \%$ had low head-circumference-for-age z (HCAZ) scores. The median (IQR) weight at birth in this study sample was $3.1(2.8 ; 3.5) \mathrm{kg}$; and the median (IQR) head circumference was $34(33 ; 35) \mathrm{cm}$.

\section{Associations between maternal psychosocial risk and offspring gestational epigenetic age acceleration at birth}

In bivariate unadjusted analyses, ethnicity $(\beta=-5.53 ; p<0.01)$, study site $(\beta=-5.23 ; p<0.01)$, offspring sex $(\beta=-3.36 ; p=0.02)$, head circumference at birth $(\beta=0.82 ; p=0.03)$, maternal trauma exposure $(\beta=8.03 ; p<0.01)$ or PTSD $(\beta=9.25 ; p<0.01)$ each was found to be significantly associated with offspring gestational EA acceleration at birth. In the final stepwise regression model, maternal trauma exposure $(\beta=7.92 ; p<0.01)$ or PTSD $(\beta=7.46$; 
$p<0.01)$ each was significantly associated with offspring gestational EA acceleration at birth (ie. the residuals of the linear model between scaled epigenetic gestational age and chronological gestational age), when controlling for ethnicity, offspring sex, head circumference at birth, maternal HIV status, and prenatal tobacco or alcohol use, Table 3. When stratifying the model by study site, these associations retained statistical significance and direction of effect, Table 3. When employing a more "narrow" definition of trauma exposure/PTSD - which adheres strictly to the DSM-5 criteria ${ }^{48}$ - maternal trauma exposure, but not PTSD, was found to be significantly associated with offspring gestational EA acceleration at birth in the full study sample $(\beta=5.54 ; p<0.01)$ and the TC Newman subset $(\beta=6.04 ; p=0.01) ;$ and tending towards significance in the Mbekweni subset $(\beta=4.94$; $p=0.06)$.

\section{DISCUSSION}

In this study of the association between maternal exposure to prenatal psychosocial risk factors and gestational epigenetic age (EA) acceleration at birth in the index offspring, we found that infants born to mothers with trauma exposure or PTSD had gestational EA acceleration versus those without such exposure, when controlling for a number of relevant covariates.

These findings are in line with emerging evidence of trauma-/PTSD-associated EA acceleration in adult combat veterans ${ }^{49,50}$. One recent meta-analysis by the Psychiatric Genomics Consortium PTSD Epigenetics Workgroup across 9 adult cohorts (combined $\mathrm{N}=2186$, including seven military samples) also reported small but significant associations each between childhood trauma exposure and severity of lifetime PTSD, and EA acceleration $^{51}$. However, neither PTSD diagnosis nor lifetime trauma exposure was associated with EA acceleration in this meta-analysis; and a small longitudinal study of male 
soldiers ( $n=96)$, assessing pre- and post-deployment, reported an inverse association between the development of PTSD symptoms and EA acceleration (although trauma was found to be significantly associated with EA acceleration in this study $)^{52}$. Further, while a small recent study ( $\mathrm{N}=101)$ reported $\mathrm{EA}$ acceleration in children exposed directly to neighbourhood violence ${ }^{53}$; EA deceleration has also been found to be associated with higher offspring distress in the context of low caregiver contact ${ }^{15}$. Thus, it may be that offspring EA acceleration or deceleration signifies exposure to prenatal maternal risk ${ }^{13}$.

A number of limitations of the current study should be acknowledged. First, the sample size was relatively small, thus decreasing the statistical power of association analyses. Nevertheless, we report important associations with trauma or PTSD exposure. Second, self-report questionnaires to assess maternal exposure to psychosocial risk factors may have resulted in under-reporting of key variables such as tobacco and/or alcohol use. Given prior evidence of associations between maternal smoking/alcohol consumption and deviations in $\mathrm{EA}^{54}$, self-reporting bias may have contributed to the lack of significant associations in our analysis. However, we have previously shown that self-reported tobacco smoking correlated well with urine cotinine measurement (an objective biomarker of tobacco smoking/exposure) in our study cohort; particularly in the Mixed ancestry sample ${ }^{23}$. Third, PTSD was not assessed using a clinician-administered diagnostic interview, which may have biased the findings.

These limitations notwithstanding, our study provides a unique preliminary exploration of associations between maternal psychosocial risk factors and offspring gestational EA acceleration at birth. To the best of our knowledge, an association between maternal trauma exposure/PTSD and gestational EA acceleration in the index offspring has not previously been reported. Future studies - incorporating larger samples sizes and/or cross-tissue analyses - are warranted in order to explore such associations further; and to delineate underlying neurobiological mechanisms. In addition, work is needed to understand the 
implications of EA acceleration at birth. For example, it is currently unknown whether EA acceleration is also associated with adverse neurodevelopmental outcomes in the affected offspring. Further, while the adverse intergenerational effects of maternal trauma exposure and PTSD have been well documented - both in the DCHS cohort ${ }^{19-21}$ and globally ${ }^{1,55}-$ the potential role of EA acceleration as a driver of these associations is yet to be determined. From a translational perspective, if EA acceleration at birth does indeed prove clinically meaningful in future, it may contribute to the development of trauma-/PTSD-specific epigenetic biomarkers to inform maternal-offspring screening, diagnostic and therapeutic interventions. 


\section{ACKNOWLEDGEMENTS}

This study was supported by the Eunice Kennedy Shriver National Institute of Child Health and Human Development of the National Institutes of Health (NICHD) under Award Number R21HD085849, the Fogarty International Center (FIC) and the Bill and Melinda Gates Foundation (OPP 1017641). The content is solely the responsibility of the authors and does not necessarily represent the official views of the National Institutes of Health.

Additional support for HJZ, DJS, NK and WB, and for research reported in this publication was by the South African Medical Research Council (SAMRC); from a Newton Advanced Fellowship (KD); and from South Africa's National Research Foundation (NRF) (HZ; grant number: 105865). WB is supported by the SAMRC National Health Scholars programme; NK and KD receive additional support from the SAMRC under Self-Initiated Research Grants. The views and opinions expressed are those of the authors and do not necessarily represent the official views of the SAMRC.

We thank the Drakenstein Child Health Study staff, and the clinical and administrative staff of the Western Cape Government Department of Health at Paarl Hospital and at the clinics for support of the study. We also thank our collaborators and students. Finally, we thank all mothers and children enrolled in the Drakenstein Child Health Study.

\section{CONFLICT OF INTEREST}

The authors on this manuscript declare no conflict of interest. 


\section{REFERENCES}

1. Shah PS, Shah J; Knowledge Synthesis Group on Determinants of Preterm/LBW Births. Maternal exposure to domestic violence and pregnancy and birth outcomes: a systematic review and meta-analyses. J Womens Health (Larchmt) 2010; 19: 20171231.

2. Testa M, Quigley BM, Eiden RD. The effects of prenatal alcohol exposure on infant mental development: a meta-analytical review. Alcohol Alcohol 2003; 38: 295-304.

3. Atwoli $L$ et al. Trauma and posttraumatic stress disorder in South Africa: analysis from the South African Stress and Health Study. BMC Psychiatry 2013; 13: 182. doi: 10.1186/1471-244X-13-182.

4. Palma-Gudiel H, Córdova-Palomera A, Eixarch E, Deuschle M, Fañanás L. Maternal psychosocial stress during pregnancy alters the epigenetic signature of the glucocorticoid receptor gene promoter in their offspring: a meta-analysis. Epigenetics 2015; 10: 893-902.

5. Rijlaarsdam J et al. An epigenome-wide association meta-analysis of prenatal maternal stress in neonates: A model approach for replication. Epigenetics 2016; 11: 140-149.

6. Horvath S. DNA methylation age of human tissues and cell types. Genome Biol 2013; 14: R115. doi: 10.1186/gb-2013-14-10-r115.

7. Hannum $\mathrm{G}$ et al. Genome-wide methylation profiles reveal quantitative views of human aging rates. Mol Cell 2013; 49: 359-367.

8. Knight AK et al. An epigenetic clock for gestational age at birth based on blood methylation data. Genome Biol 2016; 17: 206. doi: 10.1186/s13059-016-1068-z.

9. Knight AK, Conneely KN, Smith AK. Gestational age predicted by DNA methylation: potential clinical and research utility. Epigenomics 2017; Jan 23. doi: 10.2217/epi-20160157. [Epub ahead of print]. 
10. Bohlin $\mathrm{J}$ et al. Prediction of gestational age based on genome-wide differentially methylated regions. Genome Biol 2016; 17: 207.

11. Chen $\mathrm{BH}$ et al. DNA methylation-based measures of biological age: meta-analysis predicting time to death. Aging (Albany, NY) 2016; 8: 1844-1865.

12. Marioni RE et al. DNA methylation age of blood predicts all-cause mortality in later life. Genome Biol 2015; 16: 25. doi: 10.1186/s13059-015-0584-6.

13. Girchenko $P$ et al. Associations between maternal risk factors of adverse pregnancy and birth outcomes and the offspring epigenetic clock of gestational age at birth. Clin Epigenetics 2017; 9: 49. doi: 10.1186/s13148-017-0349-z. eCollection 2017.

14. Khouja JN et al. Epigenetic gestational age acceleration: a prospective cohort study investigating associations with familial, sociodemographic and birth characteristics. Clin Epigenetics 2018; 10: 86. doi: 10.1186/s13148-018-0520-1.

15. Moore SR et al. Epigenetic correlates of neonatal contact in humans. Dev Psychopathol 2017; 29(5): 1517-1538.

16. Zar HJ, Barnett W, Myer L, Stein DJ, Nicol MP. Investigating the early-life determinants of illness in Africa: the Drakenstein Child Health Study. Thorax 2015; 70: 592-594.

17. Stein DJ et al. Investigating the psychosocial determinants of child health in Africa: The Drakenstein Child Health Study. J Neurosci Methods 2015; 252: 27-35.

18. Donald KA et al. Drakenstein Child Health Study (DCHS): investigating determinants of early child development and cognition. BMJ Paediatr Open 2018; 2: e000282. doi: 10.1136/bmjpo-2018-000282. eCollection 2018.

19. Koen $\mathrm{N}$ et al. Intimate partner violence: associations with low infant birthweight in a South African birth cohort. Metab Brain Dis 2014; 29(2): 281-299.

20. Koen $\mathrm{N}$ et al. Psychological trauma and posttraumatic stress disorder: risk factors and associations with birth outcomes in the Drakenstein Child Health Study. Eur $J$ Psychotraumatol 2016; 7: 28720. doi: 10.3402/ejpt.v7.28720. eCollection 2016.

21. Koen $\mathrm{N}$ et al. Maternal posttraumatic stress disorder and infant developmental outcomes in a South African birth cohort study. Psychol Trauma 2017; 9(3): 292-300. 
22. Myers B et al. Effect of hazardous alcohol use during pregnancy on growth outcomes at birth: findings from a South African cohort study. Alcohol Clin Exp Res 2018; 42: 369377.

23. Vanker A et al. Antenatal and early life tobacco smoke exposure in an African birth cohort study. Int J Tuberc Lung Dis 2016; 20: 729-737.

24. Morin AM et al. Maternal blood contamination of collected cord blood can be identified using DNA methylation at three CpGs. Clin Epigenetics 2017; 9: 75. doi: $10.1186 / \mathrm{s} 13148-017-0370-2$.

25. Myer L, Stein DJ, Grimsrud A, Seedat S, Williams DR. Social determinants of psychological distress in a nationally-representative sample of South African adults. Soc Sci Med 2008; 66: 1828-1840.

26. Bernstein DP et al. Initial reliability and validity of a new retrospective measure of child abuse and neglect. Am J Psychiatry 1994; 151: 1132-1136.

27. Jewkes R. Intimate partner violence: causes and prevention. Lancet 2002; 359: 14231429.

28. Shamu S, Abrahams N, Temmerman M, Musekiwa A, Zarowsky C. A systematic review of African studies on intimate partner violence against pregnant women: prevalence and risk factors. PLoS One 2011; 6: e17591.doi: 10.1371/journal.pone.0017591.

29. Foa EB, Riggs DS, Dancu CV, Rothbaum BO. Reliability and validity of a brief instrument for assessing posttraumatic stress disorder. J Trauma Stress 1993; 6: 459473.

30. Stein DJ et al. DSM-5 and ICD-11 definitions of posttraumatic stress disorder: investigating "narrow" and "broad" approaches. Depress Anxiety 2014; 31: 494-505.

31. Harding TW et al. Mental disorders in primary health care: a study of their frequency and diagnosis in four developing countries. Psychol Med 1980; 10(2): 231-241.

32. Scholte WF, Verduin F, van Lammeren A, Rutayisire T, Kamperman AM. Psychometric properties and longitudinal validation of the self-reporting questionnaire (SRQ-20) in a 
Rwandan community setting: a validation study. BMC Med Res Methodol 2011; 11: 116. doi: 10.1186/1471-2288-11-116.

33. Beck AT, Ward CH, Mendelson M, Mock J, Erbaugh J. An inventory for measuring depression. Arch Gen Psychiatry 1961; 4: 53-63.

34. Beck AT, Steer RA, Garbin MG. Psychometric properties of the Beck Depression Inventory: twenty five years of evaluation. Clin Psychol Rev 1988; 8: 77-100.

35. Beck AT, Steer RA, Brown GK. Manual for the Beck Depression Inventory-II. Psychological Corporation: San Antonio, TX, 1996.

36. Cox JL, Holden JM, Sagovsky R. Detection of postnatal depression: development of the 10-item Edinburgh Postnatal Depression Scale. Br J Psychother 1987; 150: 782-786.

37. Eberhard-Gran M, Eskild A, Tambs K, Opjordsmoen S, Samuelsen SO. Review of validation studies of the Edinburgh Postnatal Depression Scale. Acta Psychiatr Scand 2001; 104: 243-249.

38. WHO ASSIST Working Group. The Alcohol, Smoking and Substance Involvement Screening Test (ASSIST): development, reliability and feasibility. Addiction 2002; 97:1183-1194.

39. Humeniuk $\mathrm{R}$ et al. Validation of the Alcohol, Smoking And Substance Involvement Screening Test (ASSIST). Addiction 2008; 103: 1039-1047.

40. van der Westhuizen C, Wyatt G, Williams J, Stein D, Sorsdahl K. Validation of the Alcohol, Smoking and Substance Involvement Screening Test in a low- and middleincome country cross-sectional emergency centre study. Drug Alcohol Rev 2016; 35: 702-709.

41. Fenton TR. A new growth chart for preterm babies: Babson and Benda's chart updated with recent data and a new format. BMC Pediatr 2003; 3: 13.

42. Fenton TR, Kim JH. A systematic review and meta-analysis to revise the Fenton growth chart for preterm infants. BMC Pediatr 2013; 13: 59. doi: 10.1186/1471-2431-13-59. 
43. World Health Organization (WHO). Physical status: the use and interpretation of anthropometry. Report of a WHO Expert Committee. World Health Organ Tech Rep Ser 1995; 854: 1-452.

44. R Core Team. R: A Language and Environment for Statistical Computing. R Foundation for Statistical Computing, 2017. https://www.R-project.org/

45. StataCorp. Stata Statistical Software: Release 14. College Station. StataCorp LP, 2015.

46. Moran S, Arribas C, Esteller M. Validation of a DNA methylation microarray for 850,000 CpG sites of the human genome enriched in enhancer sequences. Epigenomics 2016; 8: 389-399.

47. McEwen LM et al. Systematic evaluation of DNA methylation age estimation with common preprocessing methods and the Infinium MethylationEPIC BeadChip array. Clin Epigenetics. Under Review.

48. American Psychiatric Association (APA). Diagnostic and Statistical Manual of Mental Disorders, $5^{\text {th }}$ Edition. American Psychiatric Publishing: Arlington, VA, 2013.

49. Wolf EJ et al. Accelerated DNA methylation age: Associations with PTSD and neural integrity. Psychoneuroendocrinology 2016; 63: 155-162.

50. Wolf EJ et al. Accelerated DNA methylation age: Associations with posttraumatic stress disorder and mortality. Psychosom Med 2018; 80: 42-48.

51. Wolf EJ et al. Traumatic stress and accelerated DNA methylation age: A meta-analysis. Psychoneuroendocrinology 2018; 92: 123-134.

52. Boks MP et al. Longitudinal changes of telomere length and epigenetic age related to traumatic stress and post-traumatic stress disorder. Psychoneuroendocrinology 2015; 51: 506-512.

53. Jovanovic $T$ et al. Exposure to violence accelerates epigenetic aging in children. Sci Rep 2017; 7: 8962. doi: 10.1038/s41598-017-09235-9.

54. Simpkin AJ et al. Prenatal and early life influences on epigenetic age in children: a study of mother-offspring pairs from two cohort studies. Hum Mol Genet 2016; 25: 191-201. 
55. Roberts AL et al. Posttraumatic stress disorder across two generations: concordance and mechanisms in a population-based sample. Biol Psychiatry 2012; 72: 505-511. 
TABLE 1: Maternal sociodemographic characteristics and antenatal psychosocial risk

Variable

Mbekweni - n (\%) TC Newman - n (\%) Total - n (\%)

P-Value

Maternal Sociodemographic Characteristics

Number of mothers

$151(56)$

Ethnicity

Black/African

Mixed ancestry

Age at enrollment: median (IQR)

years

Primigravida

Married/cohabiting

Educational achievement

Primary education

Some secondary education

Completed secondary education

Tertiary education

Employed

Estimated household income

$<$ ZAR 1000/month

ZAR 1000 - ZAR 5000/month

$>$ ZAR 5000/month

HIV-infected

\section{Maternal Antenatal Psychosocial Risk}

Lifetime trauma exposure (MPSS)

Childhood trauma (CTQ), above

threshold

Intimate Partner Violence (IPV)

exposure

Any lifetime exposure

Any recent exposure

Stressful life events: median (IQR)

score

Psychological distress (SRQ-20):

above threshold

$149(99)$

2(1)

$27(22 ; 32)$

$49(32)$

$54(36)$

$10(7)$

$87(58)$

$45(30)$

$9(6)$

$33(22)$

$71(47)$

$60(40)$

$20(13)$

$58(38)$

$103(68)$

$52(35)$

$64(53)$

$61(50)$

$3(2)$

$118(98)$

$25(22 ; 30)$

$42(35)$

$53(44)$

11 (9)

55 (46)

$50(41)$

5 (4)

40 (33)

41 (34)

56 (46)

24 (20)

7 (6)

$69(46)$

$56(38)$

$1(1 ; 3)$

39 (26)

$62(41)$

42 (28)

42 (28)

20 (13)

14 (9)

272 (100)

152 (56)

120 (44)

$<0.01$

$26(22 ; 31)$

91 (33)

107 (39)

0.85

0.70

0.19

21 (8)

$142(52)$

95 (35)

14 (5)

73 (27)

0.14

0.04

$112(41)$

116 (43)

44 (16)

65 (24)

0.07

$<0.01$

Depression (BDI-II): above threshold

Depression (EPDS): above threshold

Alcohol exposure during pregnancy

Any tobacco use during pregnancy

(ASSIST) 
TABLE 2: Offspring anthropometry at birth

\begin{tabular}{|c|c|c|c|c|}
\hline Variable & Mbekweni - n (\%) & TC Newman - n (\%) & Total - n (\%) & P-Value \\
\hline \multicolumn{5}{|l|}{ Offspring sex } \\
\hline Male & $81(54)$ & $70(58)$ & $151(56)$ & \\
\hline Female & $70(46)$ & $51(42)$ & $121(44)$ & 0.49 \\
\hline \multicolumn{5}{|l|}{ Gestational age at birth: median } \\
\hline (IQR) weeks & $39(38 ; 40)$ & $39(38 ; 40)$ & $39(38 ; 40)$ & 0.57 \\
\hline Preterm birth & $28(19)$ & $25(21)$ & $53(19)$ & 0.66 \\
\hline Birthweight: median (IQR) kg & $3.2(2.9 ; 3.5)$ & $3.0(2.7 ; 3.4)$ & $3.1(2.8 ; 3.5)$ & 0.01 \\
\hline Low birthweight: < 2500g & $14(9)$ & $15(12)$ & $29(11)$ & 0.42 \\
\hline Birth WAZ: median (IQR) & $-0.4(-1.1 ; 0.2)$ & $-0.7(-1.3 ;-0.1)$ & $-0.5(-1.3 ;-0.01)$ & 0.03 \\
\hline Low WAZ at birth: $\leq 2$ SDs below & & & & \\
\hline mean weight-for-age & $11(7)$ & $11(9)$ & $22(8)$ & 0.61 \\
\hline $\begin{array}{l}\text { Head circumference at birth: } \\
\text { median (IQR) cm }\end{array}$ & $34(33 ; 35)$ & $34(32 \cdot 35)$ & $34(33 \cdot 35)$ & 0.07 \\
\hline Birth HCAZ: median (IQR) & $-0.5(-1.2 ; 0.4)$ & $-0.6(-1.3 ; 0.2)$ & $-0.6(-1.2 ; 0.4)$ & 0.26 \\
\hline $\begin{array}{l}\text { Low HCAZ at birth: } \leq 2 \text { SDs below } \\
\text { mean head-circumference-for-age }\end{array}$ & $19(13)$ & $14(12)$ & $33(12)$ & 0.75 \\
\hline
\end{tabular}


TABLE 3: Associations between maternal psychosocial risk factors and offspring gestational epigenetic age acceleration at birth

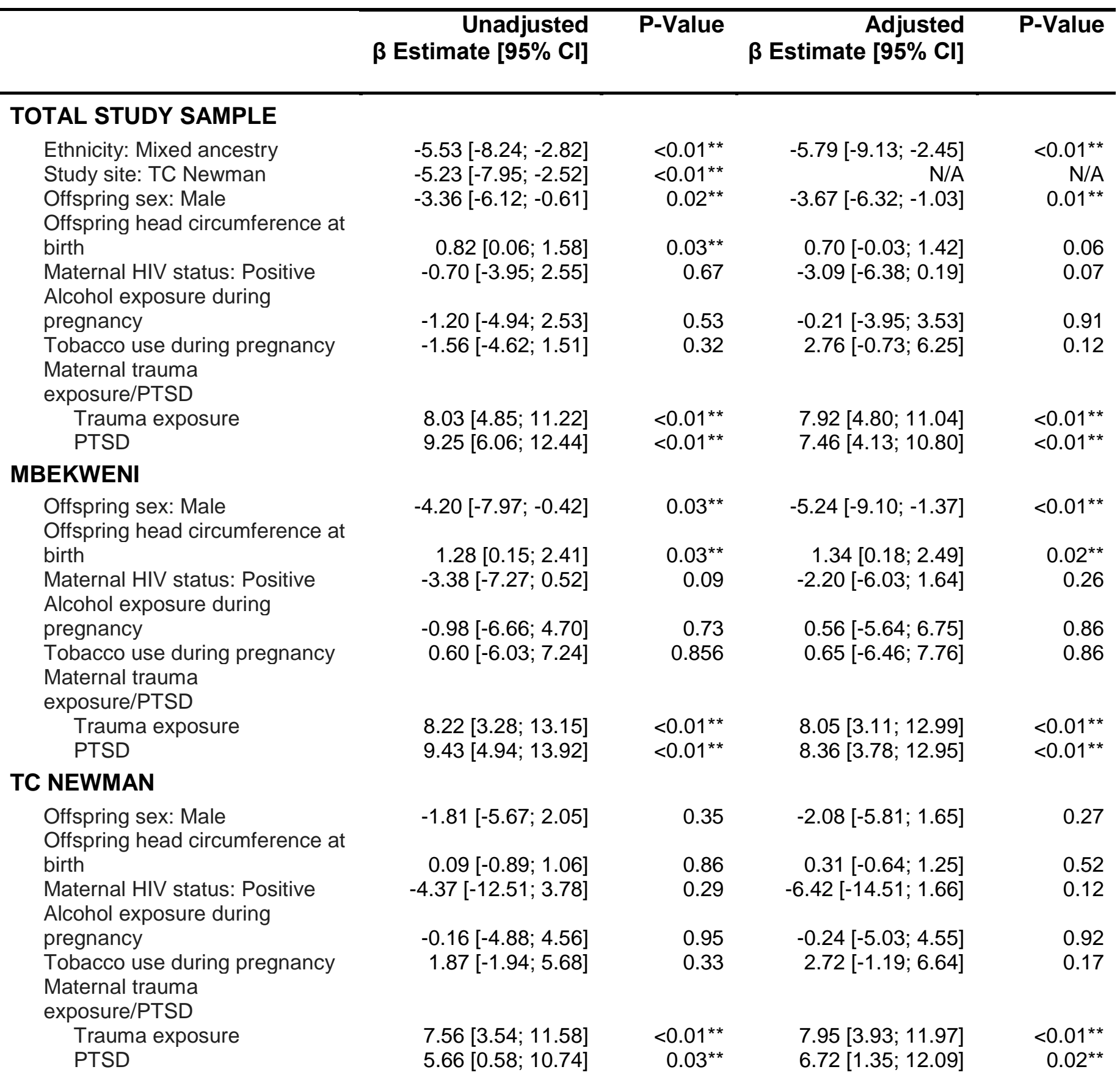

${ }^{* *} p<0.05$ 